\title{
EDITORIAL
}

\section{Myeloproliferative disorders: premalignant, stem cell, G1 diseases?}

\section{Leukemia (2006) 20, 1475-1480. doi:10.1038/sj.leu.2404295;}

Myeloproliferative disorders (MPD) are clonal hematopoietic diseases characterized by the proliferation and expansion of one or several myeloid cell lineages in the bone marrow. During the chronic phase, the cells follow their normal differentiation pathway and become mature blood cells. During a second phase, an acute syndrome may occur. The conventional classification separates MPDs in clinical entities. These include chronic myeloid leukemia $(\mathrm{CML})$, chronic neutrophilic leukemia, chronic eosinophilic leukemia, polycythemia vera (PV), essential thrombocythemia (ET) and idiopathic myelofibrosis (IMF). MPDs with different syndromes and molecular abnormalities are grouped in non-classical MPDs. Systemic mastocytosis is not classically included in MPDs but is a related disease.

Our knowledge of MPDs has made progress and now extends to several aspects of the disease, such as genes involved, classification, targeted cell, deregulated cell processes and evolution. We can answer some 'what about' questions and distinguish some main themes.

What about the targeted genes? With the development of specific therapeutical drugs, it may be more appropriate to classify MPDs according to their molecular substratum. The first description of a molecular abnormality in cancer was done in CML. An abnormal chromosome, the 'Philadelphia chromosome', was defined as a specific marker of this disease. It was later discovered that the abnormal chromosome resulted from a $t(9 ; 22)$ reciprocal translocation that creates a fusion between the $B C R$ gene at $22 q 11$ and the $A B L$ gene at $9 q 34$. This gene fusion leads to the production of the chimeric BCR-ABL oncogenic protein endowed with constitutive kinase activity. The fusion protein can be targeted by the drug imatinib mesylate, used with success for CML patients. Most molecular events characterized so far in MPDs target tyrosine kinases. Chromosomal rearrangements affect genes coding for transmembrane receptor-type (fibroblast growth factor receptor 1 (FGFR1), platelet-derived growth factor receptor $A$ and $B$ (PDGFRA), (PDGFRB)) and nonreceptor-type kinases (ABL1, janus kinase 2 (JAK2)). Point mutations target JAK2 in more than $90 \% \mathrm{PV}$, and around $50 \%$ ET and IMF. KIT is mutated in systemic mastocytosis. Unexpectedly, some hematopoietic tyrosine kinases such as FMS-like tyrosine kinase 3 (FLT3) do not seem involved in human MPDs. Based on these known molecular alterations, a preliminary molecular classification may be proposed (Table 1).

However, a good proportion of MPDs do not display obvious genetic alterations. What are the genetic abnormalities involved in these MPDs? Except for JAK2, available data from highthroughput sequencing of the human kinome have failed to evidence a kinase with a high frequency of mutations in MPDs. ${ }^{1,2}$ However, the number of MPDs studied so far may have been too limited. The search for inhibitory mutations in phosphatases and activating mutations in phosphatase inhibitors (e.g. PP1R2, PTPN11) may lead to new information. Another possibility is that kinases might be activated indirectly.

A question that arises is how can the same genetic alteration (e.g. JAK2 mutation, BCR-ABL or PCM1-JAK2 fusion) lead to different phenotypic diseases? First, additional alterations may have an impact on the cell lineage and clinical presentation of an MPD. Second, in a fusion the site of the breakpoint (e.g. $m$ or $\mathrm{M} \mathrm{bcr} \mathrm{in} \mathrm{BCR-ABL)}$ and the structure of the partner influence the resulting phenotype of the syndrome. ${ }^{3}$ Third, the strength of the kinase activation may be important. A low level of constitutive kinase activity may be generated by alteration in the kinase regulatory regions, such as JAK2 V617F and FLT3-internal tandem duplications (FLT3-ITD). The JAK2 mutation can be homozygous (20-30\%) or heterozygous. FLT3-ITD induces an MPD in mouse models, but is not found in human MPDs. A moderate level of activity may be triggered by constitutive oligomerization after gene translocation and fusion. This type of alteration is found in many different malignancies and not only in hematological diseases. Both receptor (PDGFRs, FGFR1, ALK, NTRK3, RET) and non-receptor kinases (ABL1, JAK2) are involved. Protein overexpression may also lead to moderate level of activation. Epidermal growth factor receptor, ERBB2, FGFR1 and MET are overexpressed in a number of carcinomas and other cancers. The PCM1-JAK2 protein, owing to oligomerization motifs of PCM1, may be a more active kinase than the mutated V617F JAK2 kinase. Finally, a high level of kinase activity could follow combined dimerization and alteration of a regulatory region; for example, translocation of JAK2 when the breakpoint in JAK2 interrupts the pseudo-kinase domain. Dual mutations can also occur, such as loss of regulatory domain and mutation of the kinase domain.

What about the targeted cell? Several models have been proposed to describe the origin of cancer cells. In the 'cancer stem cell model' (Figure 1), a cancer cell derives from a stem cell that has suffered one or a series of oncogenic hits. Quiescence, self-renewal, asymmetric division, prolonged lifespan and pluripotency are intrinsic features of stem cells. In regulated periods of tissue maintenance, a stem cell undergoes asymmetric division, that is, it divides in one copy of itself and a daughter cell that will enter a differentiation pathway. The first oncogenic event(s) should thus only trigger sustained cell division and prevent the stem cell to enter quiescence. This leads to unregulated expansion of stem cells and of their progeny that enters the differentiation pathway. Alternatively, in the 'progenitor model', a cancer cell may derive from a more mature proliferating progenitor cell that has de novo acquired stem cell properties, that is, self-renewal and immortality. In this case, the first oncogenic event(s) render(s) the cell able to selfrenew and to evade differentiation, apoptosis and senescence. It is not known which model predominates in human tumors, but the first model seems more parsimonious as self-renewal and survival of the progenitor cell may require more intrinsic modifications and/or signals from the environment and re-acquisition of telomere maintenance.

The BCR-ABL and BCR-FGFR1 fusion genes can be detected in cells of myeloid and B-lymphoid lineages, indicating that the targeted cell has multilineage differentiation potential. However, this cell could be a progenitor and not the stem cell. Model systems using transgenic mice have shown that BCR-ABL targets the hematopoietic stem cell. BCR-ABL alone is sufficient to induce the chronic phase of $C M L$ in transgenic mouse. BCR-ABL is not able to confer self-renewal to progenitors and must thus 
Table 1 Molecular classification of MPDs

\begin{tabular}{|c|c|c|c|c|}
\hline Class & Subclass & Molecules & Disease & Specific treatment \\
\hline \multirow[t]{3}{*}{ Mutated kinase } & JAK2 & JAK2 V617F & PV, essential thrombocythemia, idiopathic myelofibrosis & \\
\hline & KIT & KIT D816V & Systemic mastocytosis & Dasatinib \\
\hline & $A B L$ & $\mathrm{ABL} 1$ & Resistant chronic myeloid leukemia & Dasatinib, Nilotinib \\
\hline \multirow[t]{20}{*}{ Fusion kinase } & $\mathrm{ABL}$ & BCR-ABL1 & Chronic myeloid leukemia & Imatinib \\
\hline & & ETV6-ABL1 & & \\
\hline & FGFR1 & $\begin{array}{l}\text { BCR-FGFR1 } \\
\text { CEP1-FGFR1 }\end{array}$ & Atypical MPDs & \\
\hline & & FGFR1OP2-FGFR1 & & \\
\hline & & FOP-FGFR1 & & \\
\hline & & MYO18A-FGFR1 & & \\
\hline & & TIF1-FGFR1 & & \\
\hline & & ZNF198-FGFR1 & & \\
\hline & JAK2 & PCM1-JAK2 & Atypical MPDs & \\
\hline & PDGFRA & FIP1L1-PDGFRA & Chronic eosinophilic leukemia, hypereosinophilc syndrom & e Imatinib \\
\hline & & $\begin{array}{l}\text { BCR-PDGFRA } \\
\text { KIF5B-PDGFRA }\end{array}$ & & \\
\hline & PDGFRB & H4-PDGFRB & Chronic myelomonocytic leukemia, atypical MPDs & Imatinib \\
\hline & & HCMOGT1-PDGFRB & 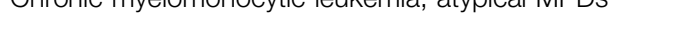 & \\
\hline & & HIP1-PDGFRB & & \\
\hline & & KIAA1509-PDGFRB & & \\
\hline & & NIN-PDGFRB & & \\
\hline & & P53BP1-PDGFRB & & \\
\hline & & PDE4DIP-PDGFRB & & \\
\hline & & RABEP1-PDGFRB & & \\
\hline & & TRIP11-PDGFRB & & \\
\hline Non-kinase & ETV6-ACS & IL3 pathway and JAK & PV & \\
\hline Non-molecularly defined yet & t ? & ? & Atypical MPDs & \\
\hline
\end{tabular}

Abbreviations: FGFR1, fibroblast growth factor receptor 1; MPD, myeloproliferative disorder; PDGFRA, platelet-derived growth factor receptor A; $\mathrm{PV}$, polycythemia vera.
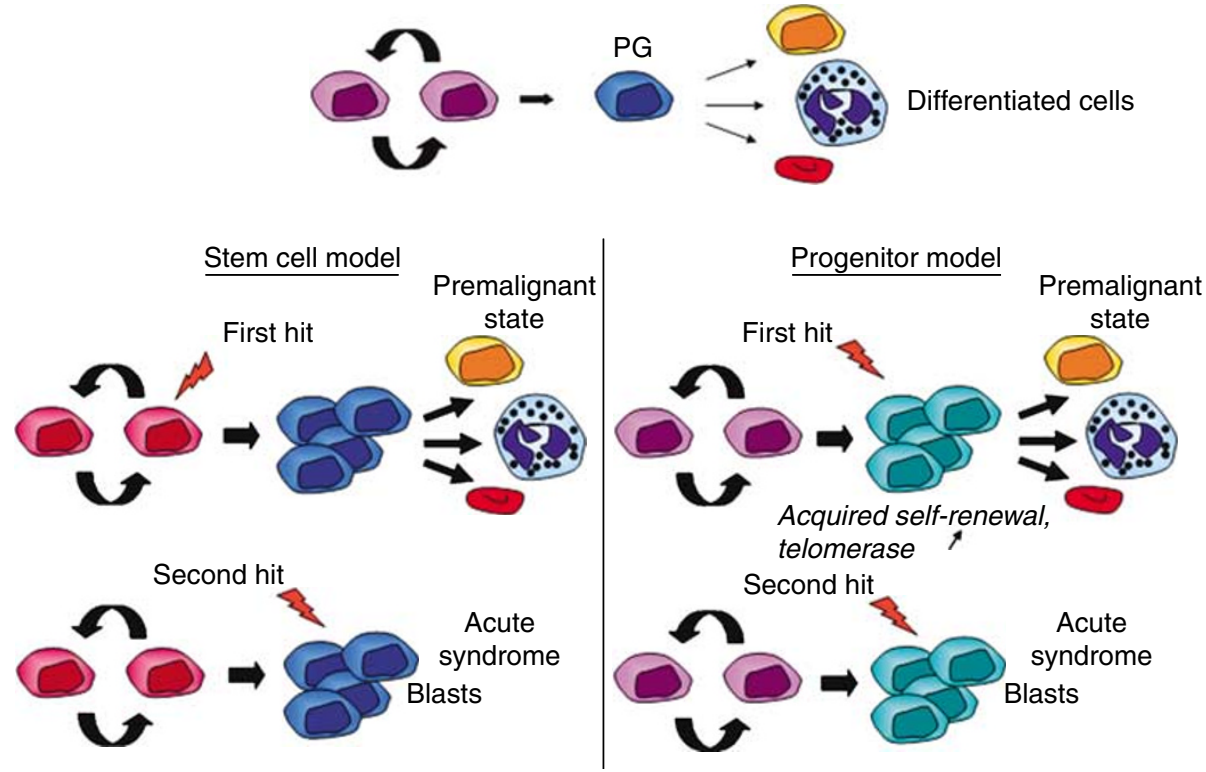

Figure 1 The cancer stem cell model. A schematic representation of a maturation pathway is represented at the top, starting with a stem cell accomplishing asymmetric division. The dividing stem cell gives birth to proliferating progenitor cells (PG), which are engaged in differentiation but still able to give rise to different types of differentiated cells. In the stem cell model, stem cells are targeted by a first hit and proliferate, but differentiation and diversity are preserved normally or almost normally. After a second hit (or series of additional mutations), which occurs either in an altered daughter cell or in the primed stem cell itself, differentiation is altered, severely or not, and an acute phase takes place. In the progenitor model, the first hit occurs in a proliferating PG that acquires self-renewal.

target the stem cell itself. ${ }^{4,5}$ Alterations involving FGFR1 are also thought to target the stem cell. A proof that oncogenic MPD kinases affect the stem cell has recently been obtained with
V617F JAK2 PV mutant. ${ }^{6}$ Stem cells and myeloid progenitors from PV patient samples were analyzed for the presence of the mutant gene. The JAK2 V617F mutation was detectable within 
both in the stem cells and in their progeny. Progenitor assays demonstrated that the differentiation potential of hematopoietic cells was already skewed toward the erythroid lineage at the stem cell level.

It is probable that malignant hematopoietic diseases may arise from both stem cells and progenitors, depending on the nature of the genetic event. Alterations that impact on proliferation and survival only, such as MPD kinases, can be tolerated by the stem cell. Their effect could be sustained asymmetric division and increased production of normal progeny. In contrast, alterations that may profoundly alter stem cell biology, for example, inducing block of differentiation and tempering with selfrenewal programs, may only target committed progenitors. Thus, a - perhaps too - schematic scenario would be that MPD kinases target stem cells, whereas chimeric transcription factors of acute leukemias (e.g. Monocytic leukemia zinc finger proteintranscriptional intermediary factor (MOZ-TIF2) or myeloid/ lymphoid or mixed-lineage leukemia-eleven-nineteen-leukemia (MLL-ENL)) target progenitors. If the latter target the stem cell or induce incomplete or abnormal re-acquisition self-renewal in progenitors, they might cause myelodysplasia. The two types of alteration and targeting (kinases/stem cells and chimeric transcription factors/progenitors) may cooperate in some circumstances. For example, if kinase genes are downstream targets of chimeric transcription factors. Cooperation could occur during the acute phase of MPD. It could be owing to secondary genetic events that take place in the progenitors and induce a block of differentiation. These secondary events could be fusions of transcription factor genes.

What about the targeted signaling pathways? A major pathway targeted by the oncogenic MPD kinases is the phosphatidylinositol-3-kinase (PI3K) pathway (Figure 2). PI3K activates the serine/threonine protein kinases AKT, which in turn activates a series of downstream effectors including target of rapamycin (TOR)/FRAP1. ${ }^{7-11}$ TOR is a central regulator of both cell growth and cell proliferation, which controls transcription, translation and ribosome biogenesis. AKT and TOR also regulate the cell cycle by controlling important regulators of the gap 1 (G1) phase of the cell cycle: cyclins, P27, retinoblastoma (RB) and MYC. The AKT/TOR pathway induces ribosomal recruitment of RNA-encoding cell cycle regulators (e.g. D-cyclins), and transcription factors (e.g. MYC, ID). Rapamycin is a specific inhibitor of TOR and arrests cells in G1. The signal from BCR$\mathrm{ABL}$ to cyclin D2 is mediated by the AKT pathway. ${ }^{12}$ The AKT pathway controls translation factors such as eukaryotic initiation factor (EIF)4E, which is also an MYC-induced protein, and phosphorylates ribosomal protein S6 kinase, which stimulates translation. The FOXO transcription factor represses D-cyclins and the G1 phase. The PI3K/AKT pathway inactivates FOXO. Coordinated entry in the $\mathrm{S}$ phase and protein synthesis lead to cell proliferation. This pathway is active in stem cells, in which MYC appears as a central regulator. ${ }^{13}$ The PI3K/AKT pathway also mediates cell survival via inactivation of proapoptotic proteins such as BAD. Proliferation and survival are the two major processes that characterize MPDs. Finally, PI3K participates to the control of centrosome duplication and to the G1 checkpoints. $^{14}$

We have recently established the gene expression profiles of $\mathrm{Ba} / \mathrm{F} 3$ cells transfected by various MPD kinases. Genes upregulated in these cells included MYC, ID1, cyclin D2, CDC25A and many ribosomal proteins (Lelièvre et al., submitted). ${ }^{15}$ Among the downregulated genes were CDKN2C and P130RBL2. A recent study has shown that the mutated JAK2 kinase also stimulates cyclin D2 and G1/S progression. $^{16}$

The PI3K/AKT pathway is altered in a large proportion of human cancers. Moreover, several tumor suppressors (phosphatase and tensin homolog (PTEN), tuberous sclerosis 1 (TSC1), TSC2, serine/theonine kinase 11 (STK11)) are negative regulators of the $\mathrm{PI} 3 \mathrm{~K}$ pathway and carry germline mutations in various

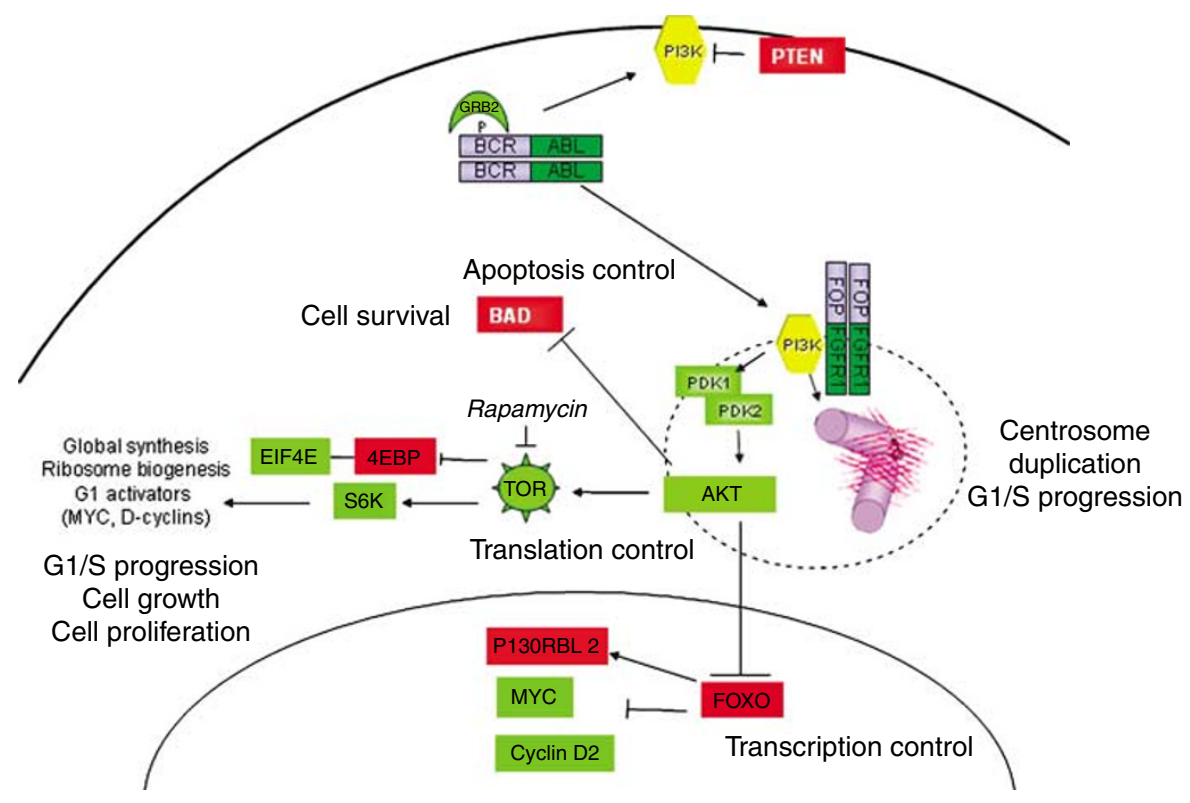

Figure 2 The PI3K/AKT/TOR pathway. Fusion tyrosine kinases such as BCR-ABL and FOP-FGFR1 (at the centrosome) activate PI3K. The signal is transmitted to AKT proteins that control several key processes, including apoptosis, transcription and translation. Transcription control is mediated via repression of FOXO factors, which activates G1 control genes such as MYC and D-cyclins. Translation control is mediated by TOR, which activates S6 kinase and eukaryotic translation factor EIF4E, turning on global protein synthesis and specific translation of G1 mRNAs. This leads to both cell growth and cell proliferation. The TOR pathway may act as a G1 checkpoint/sensor ensuring that the cell entering the cell cycle has enough size and content before engaging in division. Activators are in green and repressors are in red. 
cancer susceptibility diseases. This reinforces the view that the $\mathrm{PI} 3 \mathrm{~K}$ pathway is a major signaling cascade involved in the initiation steps of oncogenesis.

Other signaling pathways (e.g. mitogen-activated protein kinase (MAPK)) are activated by oncogenic MPD kinases. They may influence the phenotype of the disease. ${ }^{3}$ The signal transducers and activators of transcription (STAT) proteins are activated downstream of MPD kinases. Cross-talk between STAT proteins and the PI3K pathway has been demonstrated. ${ }^{17}$

What about targeted cell components? Several partners of MPD fusion kinases are centrosomal proteins (e.g. CEP1, FOP, ninein, PCM1). Some fusion kinases target the centrosome and lead to MPD. ${ }^{18}$ The centrosome is important for the regulation of cell cycle progression. It integrates various signaling pathways aimed at triggering cell division. However, centrosomal localization is not mandatory to trigger the disease. Not all fusion proteins directly target the centrosome. They may, however, target the centrosome indirectly via downstream substrates. Many signaling molecules, such as PI3K, phospholipase $C \gamma, M A P K, A K T$, are found at the centrosome, suggesting that several pathways can be deregulated at the centrosome directly or indirectly. Addressing to the centrosome may be a way for some fusion kinases to encounter a sufficient amount of PI3K substrate.

What about the targeted cell processes? The effect of an altered tyrosine kinase is the global result of its structure (including substrate binding sites), type of activation (oligomerization or mutation) and subcellular localization. Whatever the variations, we believe that a major effect of MPD kinases is on the G1 phase of the cell cycle (Figure 3). During G1 many signals are integrated; depending on the inputs, then the cell can enter in the $\mathrm{S}$ phase, pause, differentiate or die. They activate Dcyclins and other regulators of this cell cycle phase. A possible scenario of MPD initiation and evolution is therefore the following (Figure 4). The first event(s) modifie(s) the G1 phase of the cell cycle, perhaps during the asymmetric division of a stem cell. The eventual effect of the mutations targets the so-

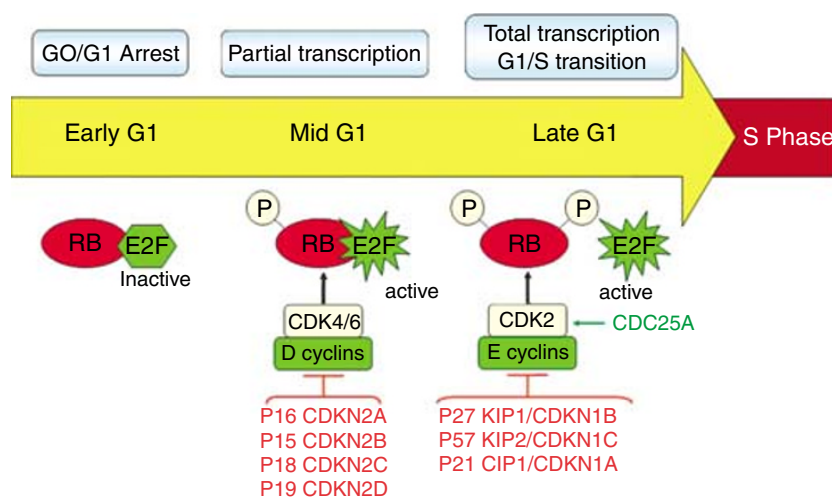

Figure 3 The G1 phase of the cell cycle. The G1 phase of the cell cycle is a key period in which a cell engaging in division begins duplicating its centrosome and builds up size and protein content. This period is a major target of MPD-activated kinases. The G1 key molecules are the pocket proteins of the RB family, which control transcription factors of the E2F family. G1 may be divided into three subperiods. In early G1, E2F are inactive owing to binding to RB. Phosphorylation of RB by cyclin D/CDK complexes in mid-G1, then by cyclin E/CDK complexes in late G1, leads to E2F release and activation. Key regulators of G1 cyclins include two families of cyclin/ CDK inhibitors (CDKN1 and CDKN2) and CDC25A phosphatase. RB, cyclins and CDKN are frequently targeted in human cancers. called 'gatekeepers' (e.g. RB). In solid tumors, mutations of PI3K pathway components, and amplification of MYC and cyclin D1 genes, and overexpression of the corresponding proteins, may also be a way to target the G1 phase. The result is sustained G1/ $S$ transition and proliferation. In MPD, this event occurs in a stem cell endowed with self-renewal and extended lifespan, and the consequence is the establishment of a premalignant state characterized by proliferation and expansion of normal progeny (Figure 4a). This creates conditions for progressive acquisition of additional mutations, aneuploidy and genetic instability. Indeed, sustained G1/S transition allows the premalignant cells to continue cycling even if centrosome abnormalities and/or DNA replication errors occur and eventually accumulate (Figure $4 \mathrm{~b}$ ). We thus propose a model in which the targeting the G1/S transition is the primary determinant of the initial stage of the disease and of subsequent genomic instability and occurrence of an acute phase. Without a forced G1/S passage,

a Normal hematopoiesis

Chronic phase

Acute phase
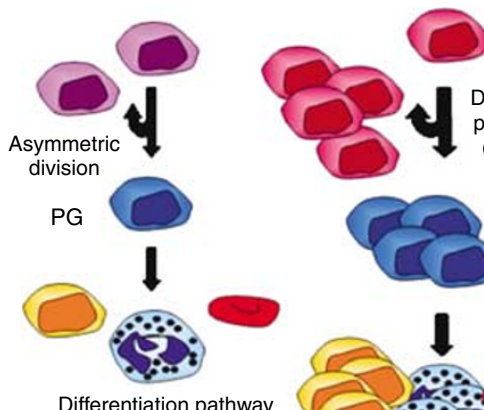

Deregulated proliferation G1 drive Accumulation

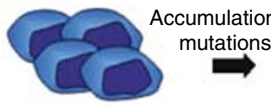

Blasts
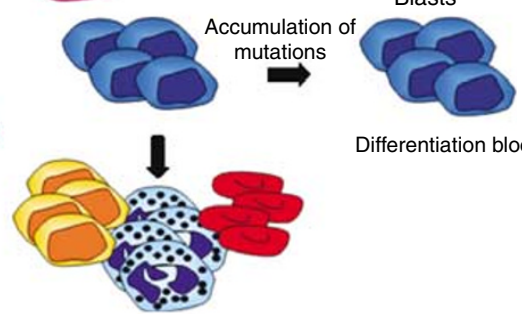

Differentiation block

b

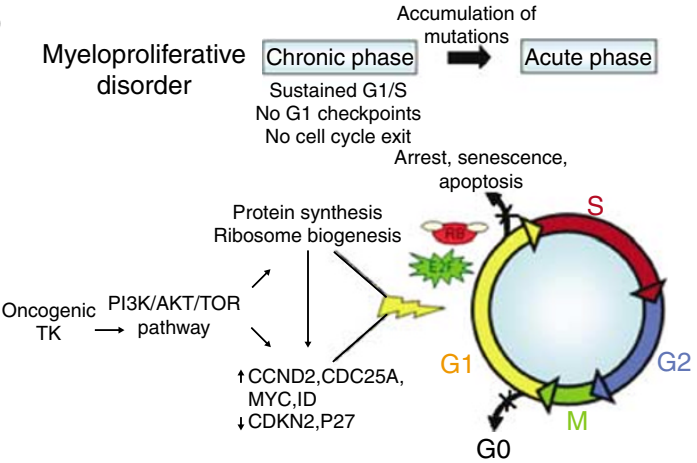

Figure 4 A model for MPD chronic and acute phase. (a) At the cell level. In normal hematopoiesis, stem cells proliferate to maintain the pool of differentiated hematopoietic cells. They do so by asymmetric division; each proliferating stem cell gives birth to a PG and to another stem cell (self-renewal) that can become quiescent or proliferate. In MPD chronic phase, after a first hit such as activation of a tyrosine kinase, sustained G1/S progression leads to expansion of the stem cell and progenitor compartments. During this unchecked phase, the differentiation pathway is grossly unaffected, but alterations in DNA and/or centrosomes accumulate. After a period of time, acute transformation occurs and is characterized by a block of differentiation (blasts). (b) At the molecular level. In the chronic (premalignant) phase, activation of a tyrosine kinase, impinges on the G1 phase of the cell cycle, mainly through the PI3K/AKT pathway. This prevents cell cycle arrest and leads to sustained cell growth and cell proliferation, but allows in the same time accumulation of alterations. When one or a series of these alterations drastically affects cell behavior, an acute syndrome replaces the chronic phase. 
a cell with mutations and/or centrosomal dysfunction would undergo cell cycle arrest or apoptosis. At the early stage, centrosome abnormalities and chromosomal instability may begin to show, but it is only when additional mutations will lead to a block of differentiation and loss of G2/M checkpoints that the acute phase will actually begin. If this hypothesis is true, a proportion of acute syndromes derived from MPD should show genetic instability, whereas others should not. Genetic targets of the acute phase could be searched among two types of genes: those controlling DNA integrity (so-called caretakers) and those able to generate aneuploidy and genetic instability. Additional genetic changes can be found in $60-80 \%$ of CML cases in blast crisis. The most frequent karyotypic abnormalities are trisomies.

Thus, in addition to the identified tyrosine kinases, proteins targeted in MPDs could be involved in the regulation of G1/S transition. This includes many potential candidates: regulators of stem cell asymmetric division (e.g. components of the $\mathrm{NOTCH}$ and WNT pathways), components of the PI3K/AKT pathway, centrosomal proteins and G1 regulators. G1 regulators are very frequently altered in human cancers, indicating that tempering with this phase may be an obligatory step on the road to cancer. ${ }^{2,19}$ However, in most cancers, other alterations and background noise blur the molecular portrait of the tumor genome. In MPDs, like probably in many other premalignant states, the importance of the alteration of the G1 phase appears more distinctly.

However, as mentioned above, many MPD cases do not show alterations of tyrosine kinases. Some of these MPDs may not be initiated by a hit in G1. Some cases show activations of the HMGA2 gene, which encodes a chromatin regulator that may not function in G1. ${ }^{20}$ These rare cases, however, are atypical MPDs with signs of myelodysplasia.

Optimal treatment of cancer lies in the targeting of specific alterations. What about the targeting drugs? In this respect, at least three approaches can be followed to treat MPDs. The first approach directly targets the actual known proteins responsible for the disease, that is, the kinases. Imatinib mesylate (Gleevec) has delivered the promise of molecularly oriented treatment. Imatinib inhibits the activated BCR-ABL fusion protein and is successfully used for CML patients. It is also active against oncogenic PDGFR and KIT kinases. However, imatinib, although it obtains durable remission of $\mathrm{CML}$, does not cure the disease and many patients acquire resistance to the antikinase, often owing to the expansion of a clone bearing additional $\mathrm{ABL}$ mutations that could not be targeted by the drug. Alternative kinase inhibitors (AMN107/nilotinib, dualspecificity SRC/ABL inhibitor dasatinib) are currently undergoing phase I/II clinical testing, with encouraging early results, $^{21,22}$ but synergistic or replacement treatments would be useful.

The second approach could target the PI3K/AKT/TOR pathway. ${ }^{11,23-27}$ Some nonspecific inhibitors of the PI3K/AKT pathway (staurosporine derivative UCN-01, alkylphospholipid perifosine, geldanamycin derivative 17-AAG) are being tested in phase II trials. For practical reasons, direct use of rapamycin is not possible in clinic. Rapamycin-derived TOR inhibitors (temsirolimus CCl-779, everolimus RAD001 and AP23573) are currently used in clinical trials. PI3K and TOR inhibitors synergize with imatinib and are attractive drugs for the treatment of MPDs and many human cancers.

As MPDs may primarily be G1 diseases, a third approach would use drugs targeting the G1 phase of the cell cycle. The CDKN2/cyclin-dependent kinase (CDK) pathway may be a good therapeutical target. Inhibitors of CDK kinases may be potent cancer drugs. ${ }^{18,28-30} \mathrm{~A}$ number of CDK inhibitors are not specific of a single CDK: butyrolactone, bryostatins, olomucine and analogs, flavopiridol, staurosporine analog UCN-01, roscovitine CYC202, paullones and purvalanol derivatives. CINK4, PD0183812 and PD0332991 inhibit CDK4/6 activity. A number of other cell cycle inhibitors have other targets, such as the proteasome (bortezomib) or histone regulation (depsipeptide, benzamide derivative), or broad activities (UCN-01).

Single-agent therapies are efficient to a certain point. Combinations of drugs that target different components of the disease (proteins, pathways, processes) should be more potent.

Viewing MPD as a premalignant disease of stem cells in which the PI3K/AKT pathway is the major driving force acting mainly on the G1/S progression is certainly too schematic. However, this view may be highly efficient, first in terms of model for other human cancers (many of the oncogenes and tumor suppressors are components of either the G1 machinery and checkpoints or the PI3K/AKT pathway), and second, in terms of therapeutical design and management. It may not be necessary to know the very details and variations of the physiopathological substratum of the disease to counter its effect; knowing the main themes could be enough.

\section{Acknowledgements}

Work on this topic in our laboratory is supported by Institut PaoliCalmettes, Inserm, and grants from Institut National du Cancer/ Cancéropôle $(\mathrm{ACl} 2005)$ and the Association Laurette Fugain (2005).

H Lelièvre $^{1}$, A Ferrand ${ }^{1}$, M-J Mozziconacci ${ }^{1}$, D Birnbaum $^{1}$ and B Delaval ${ }^{2}$

${ }^{1}$ Laboratoire d'Oncologie Moléculaire, Centre de Recherche en Cancérologie de Marseille, UMR599 Inserm et Institut Paoli-Calmettes, Marseille, France and

${ }^{2}$ University of Massachusetts Medical School/Biotech, Worcester, Massachusetts, USA

E-mail: birnbaum@marseille.inserm.fr

\section{References}

1 Futreal PA, Coin L, Marshall M, Down T, Hubbard T, Wooster R et al. A census of human cancer genes. Nat Rev Cancer 2004; 4: 177-183.

2 http://www.sanger.ac.uk.

3 Roumiantsev S, Krause DS, Neumann CA, Dimitri CA, Asiedu F, Cross NC et al. Distinct stem cell myeloproliferative/T lymphoma syndromes induced by ZNF198-FGFR1 and BCRFGFR1 fusion genes from 8p11 translocations. Cancer Cell 2004; 5: $287-298$.

4 Jamieson $\mathrm{CH}$, Ailles LE, Dylla SJ, Muijtjens $M$, Jones C, Zehnder JL et al. Granulocyte-macrophage progenitors as candidate leukemic stem cells in blast-crisis CML. N Engl J Med 2004; 351: 657-667.

5 Huntly BJ, Shigematsu H, Deguchi K, Lee BH, Mizuno S, Duclos N et al. MOZ-TIF2, but not BCR-ABL, confers properties of leukemic stem cells to committed murine hematopoietic progenitors. Cancer Cell 2004; 6: 587-596.

6 Jamieson CH, Gotlib J, Durocher JA, Chao MP, Mariappan MR, Lay $\mathrm{M}$ et al. The JAK2 V617F mutation occurs in hematopoietic stem cells in polycythemia vera and predisposes toward erythroid differentiation. Proc Natl Acad Sci USA 2006; 103: 6224-6229.

7 Liang J, Slingerland JM. Multiple roles of the PI3K/PKB (Akt) pathway in cell cycle progression. Cell Cycle 2003; 2: 339-345.

8 Bader AG, Kang S, Zhao L, Vogt PK. Oncogenic PI3K deregulates transcription and translation. Nat Rev Cancer 2005; 5: 921-929.

9 Kharas MG, Fruman DA. ABL oncogenes and phosphoinositide 3kinase: mechanism of activation and downstream effectors. Cancer Res 2005; 65: 2047-2053. 
10 Samuels Y, Ericson K. Oncogenic PI3K and its role in cancer. Curr Opin Oncol 2006; 18: 77-82.

11 Martelli AM, Nyakern M, Tabellini G, Bortul R, Tazzari PL, Evangelisti $\mathrm{C}$ et al. Phosphoinositide 3-kinase/Akt signaling pathway and its therapeutical implications for human acute myeloid leukemia. Leukemia 2006; 20: 911-928.

12 Deininger MW, Vieira SA, Parada Y, Banerji L, Lam EW, Peters G et al. Direct relation between BCR-ABL tyrosine kinase activity and cyclin D2 expression in lymphoblasts. Cancer Res 2001; 61: 8005-8013.

13 Moore KA, Lemischka IR. Stem cells and their niches. Science 2006; 311: 1880-1885.

14 van Opstal A, Bijvelt JJ, Margadant C, Boonstra J. Role of signal transduction and actin in G1 phase progression. Adv Enzyme Regul 2005; 45: 186-200.

15 Lelièvre $\mathrm{H}$, Cervera $\mathrm{N}$, Finetti $\mathrm{P}$, Delhommeau F, Vainchenker W, Bertucci $\mathrm{F}$ et al. Oncogenic kinases of myeloproliferative disorders induce both protein synthesis and G1 activators. Submitted.

16 Walz C, Crowley BJ, Hudon HE, Gramlich JL, Neuberg DS, Podar $\mathrm{K}$ et al. Activated JAK2 with the $\mathrm{V} 617 \mathrm{~F}$ point mutation promotes G1/S-phase transition. J Biol Chem 2006, 21 April [E-pub ahead of print].

17 Nyga R, Pecquet C, Harir N, Gu H, Dhennin-Duthille I, Regnier A et al. Activated STAT5 proteins induce activation of the PI 3kinase/Akt and Ras/MAPK pathways via the Gab2 scaffolding adapter. Biochem J 2005; 390: 359-366.

18 Delaval B, Lelièvre H, Birnbaum D. Myeloproliferative disorders: the centrosome connection. Leukemia 2005; 19: 1739-1744.

19 Malumbres M, Barbacid M. To cycle or not to cycle: a critical decision in cancer. Nat Rev Cancer 2001; 1: 222-231.
20 Odero MD, Grand FH, Iqbal S, Ross F, Roman JP, Vizmanos JL et al. Disruption and aberrant expression of HMGA2 as a consequence of diverse chromosomal translocations in myeloid malignancies. Leukemia 2005; 19: 245-252.

21 Deininger $M$. Resistance to imatinib: mechanisms and management. J Natl Compr Cancer Network 2005; 3: 757-768.

22 Shah NP. Loss of response to imatinib: mechanisms and management. Hematology (Am Soc Hematol Educ Program) 2005, 183-187.

23 Asnaghi L, Bruno P, Priulla M, Nicolin A. mTOR: a protein kinase switching between life and death. Pharmacol Res 2004; 50: 545-549.

24 Chan S. Targeting the mammalian target of rapamycin (mTOR): a new approach to treating cancer. Br J Cancer 2004; 91: 1420-1424.

25 Amaravadi R, Thompson CB. The survival kinases Akt and Pim as potential pharmacological targets. J Clin Invest 2005; 115: 2618-2624.

26 Stephens L, Williams R, Hawkins P. Phosphoinositide 3-kinases as drug targets in cancer. Curr Opin Pharmacol 2005; 5: 357-365.

27 Cortot A, Armand JP, Soria JC. PI3K-AKT-mTOR pathway inhibitors. Bull Cancer 2006; 93: 19-26.

28 Collins I, Garrett MD. Targeting the cell division cycle in cancer: CDK and cell cycle checkpoint kinase inhibitors. Curr Opin Pharmacol 2005; 5: 366-373.

29 Ortega S, Malumbres M, Barbacid M. Cyclin D-dependent kinases, INK4 inhibitors and cancer. Biochim Biophys Acta 2002; 1602: 73-87.

30 Schwartz GK, Shah MA. Targeting the cell cycle: a new approach to cancer therapy. J Clin Oncol 2005; 23: 9408-9421. 